\title{
Optimal decision decoding algorithm (ODDA) for an asymmetric TWRC system
}

\author{
Siamack Ghadimi \\ Department of Electrical and Computer Engineering, Western University, London, Ontario, N6A 3K7, Canada
}

Received September 02, 2019; Revised October 8, 2019; Accepted October 30, 2019

Copyright (C) 2020 by authors, all rights reserved. Authors agree that this article remains permanently open access under the terms of the Creative Commons Attribution License 4.0 International License

\begin{abstract}
The relay network has many advantages, such as reliability against failures, and offers redundancy, selfconfiguring, and self-healing. The purpose of his paper is to simulate an Asymmetric Modulation (AM) for wireless Two Way Relay Channel (TWRC) system in the presence of additive white Gaussian noise (AWGN) to compute Bit Error Rate (BER) performance of transmitted data. Finally, by utilizing a novel algorithm, which named Optimal Decision Decoding Algorithm (ODDA) the BER has decreased, and performance of the system improved.
\end{abstract}

Keywords Two Way Relay Channel, Network coding, Digital Network Coding, Analogue Network Coding, Algorithm, Asymmetric Modulation

\section{Introduction}

The wireless Two Way Relay Channel (TWRC) system is based on Network coding, which is a way of relaying packets of information, that are received from different nodes, by combining them together and mapping for broadcasting. The idea of network coding is not new and has existed for more than a decade and proposed in [1]. However, the application of network coding has been shown to increase the network throughput. In general there are two interfaces of network coding [2]: Digital Network Coding (DNC): Nodes transmit sequentially, relay receives and demodulates signals and then does a linear combination, e.g. XOR-ing, of received data for broadcasting to respective nodes. It takes 3 time slots. Sometimes the schedule calls for traditional multihop transmissions.

Analogue Network Coding (ANC): Nodes send signals simultaneously to the relay (Multiple Access Channel (MAC) phase), signals combine in the physical channel, called superimposed, and then the relay jointly decodes (map) the superimposed signal. Lastly, the relay re-encodes the signal to an optimized binary signal for broadcasting (Broadcast Channel (BC) phase) to respective nodes [3], Figure 1.

The ANC scheme improves the throughput by taking one less time slot as compared to its DNC counterpart. It operates in half duplex mode, but requires strict synchronization [3, 4] in symbol-phase and carrier-frequency between nodes.

The network coding interface, discussed in this paper is analogue network coding.

The rest of the paper is organized as follows. The next section discusses the overview of the system model for Two Way Re-

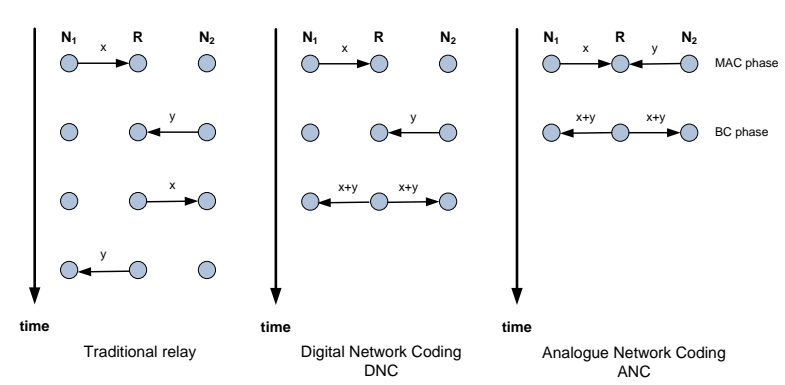

Figure 1. Network coding

lay Channel (TWRC) system, while section 3 describes Error probability analysis for TWRC systems. Section 4 presents adaptive coding and modulation for TWRC systems, meanwhile, section 5 presents a novel algorithm, named Optimal Decision Decoding Algorithm (ODDA), and at last, section 6 presents simulation and results of the ODDA algorithm. Finally, this paper is concluded in section 7 .

\section{System model}

Consider a TWRC system with 1 relay node $\mathbf{R}$ and two terminal nodes $\mathbf{N}_{\mathbf{i}}, i=1,2$ shown in Figure 2, [1, 2].

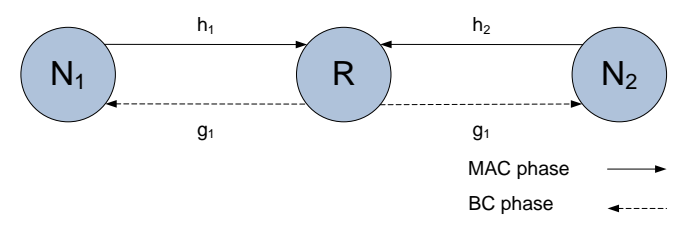

Figure 2. System Model for a Two Way Relay Channel

Let $S_{i}$ denote the message from the source nodes $N_{i}$ for $i=[1,2], S_{i} \sim \mathcal{C N}(0, P)$.

MAC phase: MAC phase means that when $N_{1}$ and $N_{2}$ simultaneously sent their message signal to the relay $R$, which they respectively are:

$$
\begin{aligned}
& r_{1}=h_{1} \sqrt{E_{1}} S_{1}(n)+w_{r}(n) \\
& r_{2}=h_{2} \sqrt{E_{2}} S_{2}(n)+w_{r}(n)
\end{aligned}
$$

where $h_{1}$ and $h_{2}$ independent complex channel fading coefficient between $N_{i}$ and $R$, i.e. $h_{i} \backsim \mathbf{C N}\left(0, \sigma^{2}\right)$. Assume that the channel is unknown to transmitting nodes but perfectly known 
at receiving nodes. $E_{1}$ and $E_{2}$ are transmission energies from nodes $N_{1}$ and $N_{2}$ respectively. $w_{r}(n)$ is additive white Gaussian noise (AWGN) with mean zero (no dc) and variance $\sigma^{2}$.

For simplicity, it can be assumed that channel coefficients are constant in two consecutive time slots. It is assumed that the phase of the transmitted signals from $N_{1}$ and $N_{2}$ are synchronized at the relay node.

At the relay these two synchronized signals add up together $r=r_{1}+r_{2}$ to form a superimposed signal as:

$$
r(n)=h_{1} \sqrt{E_{1}} S_{1}(n)+h_{2} \sqrt{E_{2}} S_{2}(n)+w_{r}(n)
$$

Now if the relay amplifies $r(n)$ by a factor $\beta$ and broadcast $\beta r(n)$ to nodes, the received signal at $N_{1}$ is given by [2]

$$
\begin{gathered}
\beta r_{s_{i}}(n)=\beta h_{1}^{2} \sqrt{E_{1}} S_{1}(n)+\beta h_{1} h_{2} \sqrt{E_{2}} S_{2}(n) \\
+\beta h_{1} w_{r}(n)+w_{r}(n)
\end{gathered}
$$

But in the network coding, first the relay detects the received signal, then modulates and broadcasts the detected signal to the nodes. The detection is done by using full channel state information and soft decision decoding. The Maximum Likelihood (ML) detection is given by:

$$
d=\min _{S_{i}}\left\|y_{i}-h_{1} \sqrt{E_{1}} S_{1}-h_{2} \sqrt{E_{2}} S_{2}\right\|^{2} \quad i=[1,2]
$$

The solution for this equation will be uniquely decided, since it is considered that fading channels are totally independent of each other.

The detected signal is mapped by XOR-ed bits from two nodes at the relay.

$$
\hat{b}_{x}=\hat{b}_{i} \oplus \hat{b}_{j} \quad i, j=[1,2] \quad i \neq j
$$

Broadcast phase: Broadcast means the relay converts back bits $b_{x}$ into symbols and broadcasts to the nodes $N_{1}$ and $N_{2}$.

$$
y_{s_{i}}=g_{i} \cdot \sqrt{E_{r}} X+w_{r}(n) \quad i=[1,2]
$$

were $y_{s_{i}}$ is the signal sent by the relay to $N_{1}$ and $N_{2}$. $\mathrm{X}$ is the superimposed symbol message at the relay. $g_{i}, i=[1,2]$ are the complex channel gains from the relay to nod $N_{1}$ and $N_{2}$ respectively, and $\sqrt{E_{r}}$ is the transmission energy at the relay, see Figure 2. lows:

By applying the minimum Euclidean distance rule, it fol-

$$
d_{i}=\min _{X}\left\|y_{s_{i}}-g_{i} \sqrt{E_{r}} X\right\|^{2} \quad i=[1,2]
$$

Finally as the nodes saved their own transmitted signals $b_{j}$ for $j=[1,2]$, they simply extracted the transmitted signal from the counterpart node by XOR-ing their own signal from the received signal.

$$
\hat{\hat{b}}_{i}=\hat{\hat{b}}_{x} \oplus b_{j} \quad i, j=[1,2] \quad i \neq j
$$

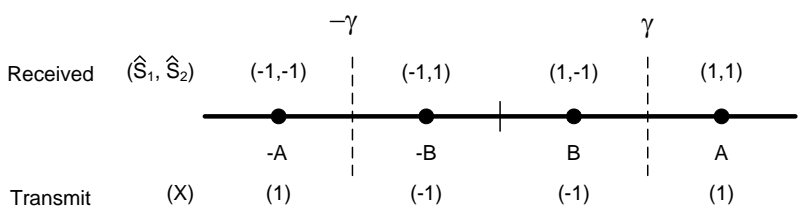

Figure 3. Constellation of received signal at the relay node where $A=|| h_{1}\left|\sqrt{E_{1}}+\right| h_{2}\left|\sqrt{E_{2}}\right|$ $B=|| h_{1}\left|\sqrt{E_{1}}-\right| h_{2}\left|\sqrt{E_{2}}\right|$

$S_{i} \in\{1,-1\}$ for $i=[1,2]$ denotes its symbol information. In this system, it is assumed $\left|h_{1}\right|>\left|h_{2}\right|$ that the constellation of the received signal $r$ at relay becomes the same as Figure 3, [5].

Where $\gamma$ is the decision boundary to map $X=2 \hat{b}_{x}-1$ which is the symbol information, the relay transmits to both $N_{1}$ and $N_{2}$ during the broadcast phase. When $\left(S_{1}, S_{2}\right)$ is equal to $[-1,1]$ or $[1,-1]$, it will fall into the decision region $[-\gamma, \gamma]$ and hence the relay will transmit -1 towards both sources. Again when $\left(S_{1}, S_{2}\right)$ is equal to $[1,1]$ or $[-1,-1]$, it will fall into the decision region outside of $[-\gamma, \gamma]$ and the relay will transmit 1 towards both sources.

To minimize bit errors, the optimal boundary of $\gamma$ is given by

$$
\begin{aligned}
& e^{-(\gamma-A)^{2} / 2 \sigma_{n}^{2}}+e^{-(\gamma+A)^{2} / 2 \sigma_{n}^{2}}=e^{-(\gamma-B)^{2} / 2 \sigma_{n}^{2}} \\
& +e^{-(\gamma+B)^{2} / 2 \sigma_{n}^{2}}
\end{aligned}
$$

which is equal to

$$
\frac{\cosh \left(\frac{\gamma A}{\sigma_{n}^{2}}\right)}{\cosh \left(\frac{\gamma B}{\sigma_{n}^{2}}\right)}=e^{\left(A^{2}-B^{2}\right) / 2 \sigma_{n}^{2}}
$$

For high SNRs where both A and B are greater than $3 \sigma_{n}^{2}$, the value of $\gamma$ will approach $\frac{A+B}{2}$ as the decision boundary. The average BEP at the relay $P_{r}$ for the MAC phase is written by

$$
\begin{aligned}
& P_{r}\left(\hat{b}_{x} \neq b_{x} \mid y_{r}(n)\right)=E_{\lambda_{\min }}\left[Q\left(\lambda_{\min }\right)\right] \\
& +\frac{1}{2} E_{\lambda_{\min }, \lambda_{\max }}\left[Q\left(2 \lambda_{\max }-\lambda \min \right)\right] \\
& -\frac{1}{2} E_{\lambda_{\min }, \lambda_{\max }}\left[Q\left(2 \lambda_{\max }+\lambda \min \right)\right]
\end{aligned}
$$

where

$$
\begin{aligned}
& \lambda_{\min }=\min \left(\left|h_{1}\right| \sqrt{\frac{2 E_{1}}{2 \sigma_{n}^{2}}},\left|h_{2}\right| \sqrt{\frac{2 E_{2}}{2 \sigma_{n}^{2}}}\right) \\
& \lambda_{\max }=\max \left(\left|h_{1}\right| \sqrt{\frac{2 E_{1}}{2 \sigma_{n}^{2}}},\left|h_{2}\right| \sqrt{\frac{2 E_{2}}{2 \sigma_{n}^{2}}}\right)
\end{aligned}
$$

where $E_{x}[$.$] denotes the expectation operation over random$ variable $x$. By simplify this equation by approximating it with the dominant Q-function (Q-function is the tail probability of the standard normal distribution.) term, we get:

\section{Error probability analysis}

MAC phase: The BPSK modulated signal of $N_{i}$ will then be $S_{i}=2 b_{i}-1$ where $b_{i} \in\{0,1\}$ denotes binary information, and 


$$
P_{r} \approx E_{\lambda_{\text {min }}}\left[Q\left(\lambda_{\text {min }}\right)\right]=\frac{1}{2} \times\left(1-\sqrt{\frac{E\left[\left|h_{1}\right|^{2}\right] \cdot E\left[\left|h_{2}\right|^{2}\right] \cdot \frac{E_{1}}{2 \sigma_{n}^{2}} \cdot \frac{E_{2}}{2 \sigma_{n}^{2}}}{E\left[\left|h_{1}\right|^{2}\right] \cdot E\left[\left|h_{2}\right|^{2}\right] \cdot \frac{E_{1}}{2 \sigma_{n}^{2}} \cdot \frac{E_{2}}{2 \sigma_{n}^{2}}+E\left[\left|h_{1}\right|^{2}\right] \cdot \frac{E_{1}}{2 \sigma_{n}^{2}}+E\left[\left|h_{2}\right|^{2}\right] \cdot \frac{E_{2}}{2 \sigma_{n}^{2}}}}\right)
$$

Broadcast phase: The average BEP at the node $N_{i}, i=$ $[1,2]$ are given in [6] which are

$$
\begin{aligned}
& P_{N_{i}}\left(\hat{\hat{b}}_{x} \neq \hat{b}_{x} \mid y_{N_{i}}(n)\right)=E_{h_{i}}\left[Q\left(\left|h_{i}\right| \sqrt{\frac{2 E_{r}}{2 \sigma_{n}^{2}}}\right)\right] \\
& =\frac{1}{2}\left(1-\sqrt{\frac{\frac{E_{i}}{2 \sigma_{n}^{2}} \cdot E_{r}}{2 \sigma_{n}^{2}+\frac{E_{i}}{2 \sigma_{n}^{2}} \cdot E_{r}}}\right) \quad i=[1,2]
\end{aligned}
$$

End-to-end bit error probability: The end-to-end BEP from node- 1 to node- 2 is defined as the BEP between the signal transmitted by node- 1 and the signal decoded at node- 2 given by

$$
\begin{aligned}
& P_{1 \rightarrow 2}=P\left(\hat{\hat{b}}_{1} \neq b_{1}\right) \\
& =1-\left(1-P_{r}\right)\left(1-P_{N_{2}}\right)-P_{r} P_{N_{2}} \\
& =P_{r}+P_{N_{2}}-2 P_{r} P N_{2} \\
& P_{2 \rightarrow 1}=P\left(\hat{\hat{b}}_{2} \neq b_{2}\right)=P_{r}+P_{N_{1}}-2 P_{r} P N_{1}
\end{aligned}
$$

Finally, the overall instantaneous end-to-end BEP for the given channel gain is

$$
P_{\text {inst }}=\frac{1}{2}\left(P_{1 \rightarrow 2}+P_{2 \rightarrow 1}\right)
$$

\section{Adaptive Coding and Modulation}

For transmission of high priority data, it is more important to have a reliable link than a higher throughput link. Furthermore, sometimes it is advantageous to have a higher throughput link when the channel condition is good and priority is not important. The $\mathrm{ACM}^{1}$ can get the information about data priority via coded data in the received signal, and the information about channel condition via Channel State Information (CSI) by using a pilot or embedded pilot symbol in the received signal. Therefore, in this part Asymmetric Modulation (AM) is applied in the TWRC system and performance of the scheme is investigated. Here a typical case has been chosen, where one source node uses antipodal BPSK modulation, while the other one uses orthogonal QPSK modulation scheme [7], Figure 4. The MAC transmission is the same as the symmetric scenario, but broadcast transmission will be accomplished by two sequenced BPSK transmissions to transfer a QPSK signal from node- 2 to node- 1 . Of course, in this phase node- 2 will get same signal twice.

The relay's constellation regions are obtained by calculation of channel gain $h 1$ and $h 2$, which means de-noising mapping will evaluate according to the channel gain. The received signal has 8 ideal normalized constellation points, Figure 5.

\footnotetext{
${ }^{1}$ Adaptive Coding and Modulation (ACM) is a technique, which automatically change the modulation depend on priority of data or channel condition.
}

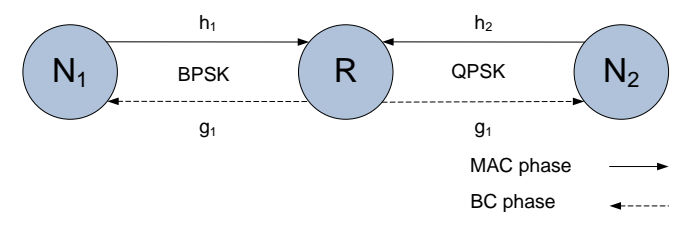

Figure 4. An asymmetric TWRC system

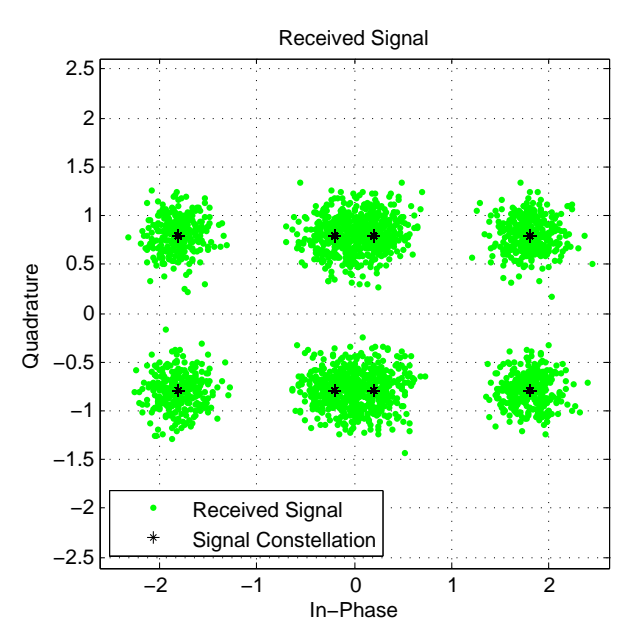

Figure 5. Constatation at relay for asymmetric TWRC BPSK and QPSK modulation

\section{Optimal decision decoding algorithm (ODDA) for asymmetric TWRC}

In this section a novel algorithm based on soft decision method proposed for improvement of received signal at one node in an asymmetric TWRC, which named Optimal Decision Decoding Algorithm (ODDA).

To present the proposed algorithm, the same system used as in the previous section, a QPSK and BPSK asymmetric TWRC system. The symbols for BPSK and QPSK modulations are typically given by $S_{i B P S K}=\{ \pm 1\}$ and $S_{i Q P S K}=\{ \pm 1 \pm j\}$ respectively.

As mentioned before, in an asymmetric TWRC system there are two broadcast transmission from the relay in return for one MAC transmission, and the number of sequenced broadcasts depends on the modulation technique. As we know in this example the TWRC system needs two sequenced broadcasts to accomplish a complete transmission.

In conventional TWRC system, received signals at relay after decision and de-noise will be modulated and broadcasted. But in the proposed algorithm, after decision and de-noise, one of the signals will be formed by XOR-ing of decoded bits of the complex received signal and the other one will be formed the same as the conventional TWRC model. In node-1 after decision, QPSK bits from node-2 will be recovered by XOR-ing. But in node-2, in the first step uses hard decision method to measures Euclidean distance of two sequenced received signals. In the next step decision rules applies to the 
signal which have least Euclidean distance. This results in decision with the highest probability for received signal, see the algorithm below 1 .

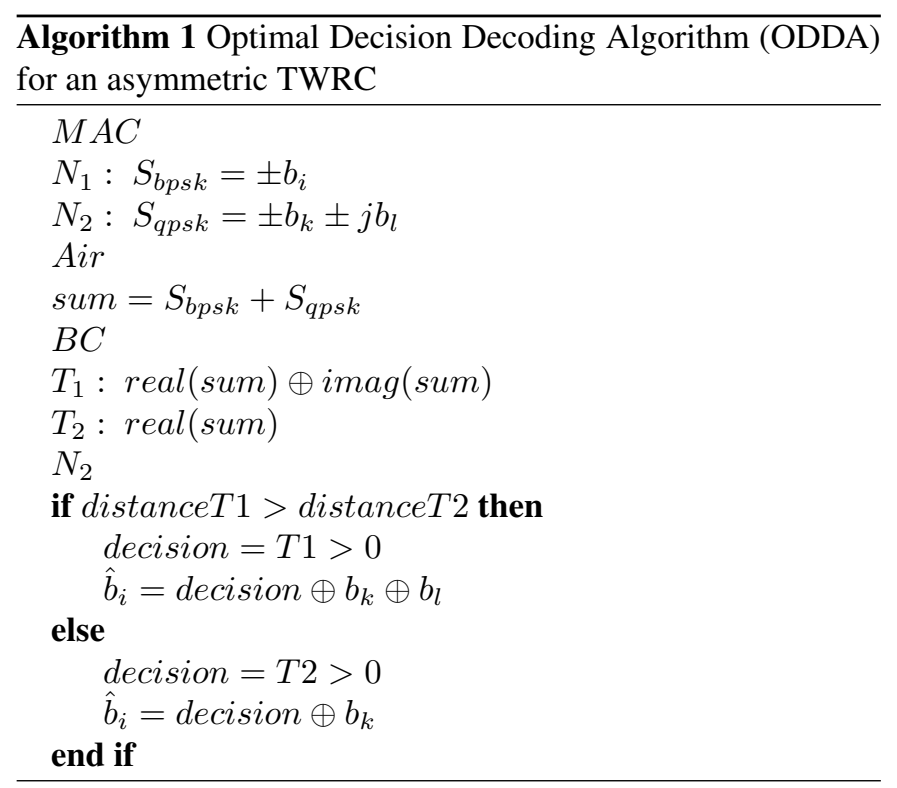

This method allows the node- 2 to detect a limited number of errors that occur anywhere in the signal and correct these errors without re-transmission or cost.

\section{Simulations and results}

In this section, a performance evaluation will be shown for a wireless TWRC system under a Rayleigh fading conditions with AWGN environments. The simulation is done by generation of uncorrelated Rayleigh fading sequences by using the simulation software MATLAB. The MATLAB random number generator produce normally distributed random numbers. Based on modulation technique and detection method in a loop computes the number of bit errors and bit error rate.

In all the following steps the relay will amplify the received signal by a factor of $\beta$ before broadcasting. The amplification factor $\beta$ has been chosen as [8]:

$$
\beta=\sqrt{\frac{P_{R}}{\left|h_{1}\right|^{2} \cdot P_{N_{1}}+\left|h_{2}\right|^{2} \cdot P_{N_{2}}+\sigma^{2}}}
$$

where $\sigma^{2}$ is AWGN variance. $P_{R}, P_{N_{1}}$ and $P_{N_{2}}$ are average transmission power at the relay and at the node 1 and at the node 2 respectively.

For BPSK modulation at $N_{1}$, symbols is the same as Section 3, but for QAM modulation at $N_{2}$, the symbols will then be $\left(2 b_{k}-1\right)+j\left(2 b_{l}-1\right)$ where $b_{k}, b_{l} \in\{0,1\}$ denotes binary information, and $S_{i} \in\{ \pm 1 \pm j\}$ for $i=[1,2,3,4]$ denotes its symbol information.

It has assumed both channels are affected by Rayleigh fading with AWGN and $\left|h_{2}\right|>\left|h_{1}\right|$.

Figure 6 represents the performance graphs of the asymmetric modulation of a TWRC system for the relay and both nodes, as well as performance of the symmetric BPSK TWRC system.

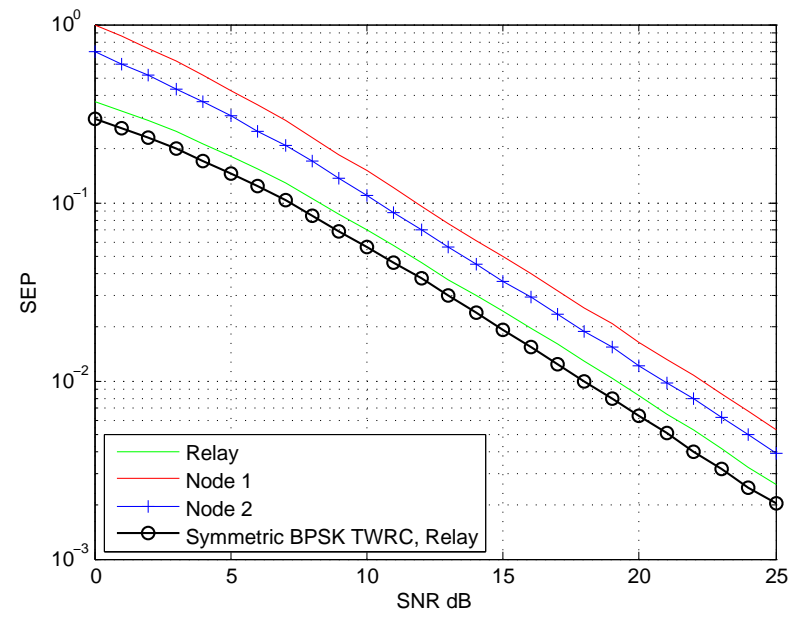

Figure 6. Asymmetric modulation of TWRC

The performance of symmetric transmission is better than asymmetric, because the decision boundaries of the asymmetric Euclidean distance points on constellation are closer to one another compared to the decision boundaries in the symmetric constellation, see Figure 3 and Figure 5.

For accurate comparison between two nodes, It has assumed $\left|h_{1}\right|=\left|h_{2}\right|$, see Figure 7. As this figure shows, the BPSK signal received by node- 2 has better performance than the QPSK signal received by node-1, and this is because the QPSK signal received from node- 2 at the relay should broadcast two sequenced transmissions, which at the end decrease performance of the QPSK symbol compared to the BPSK symbol.

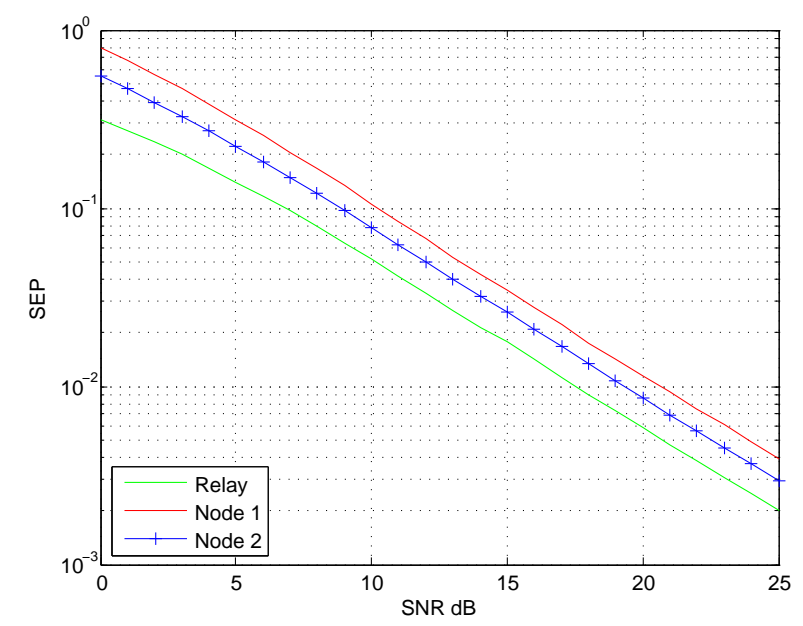

Figure 7. Asymmetric modulation of TWRC when $\left|h_{1}\right|=\left|h_{2}\right|$

It is easy from Figure 8 to see that, after the implementation of the optimal decision decoding algorithm, the performance curve of the received signal in node- 2 has been improved by around $1 \mathrm{~dB}$. The performance for node- 1 remains the same. The same algorithm may simply apply to higher order modulations in an asymmetric TWRC system.

\section{Conclusions}

In this paper the performance of a wireless Two Way Relay Channel (TWRC) system investigated by utilizing a novel algorithm named Optimal Decision Decoding Algorithm 


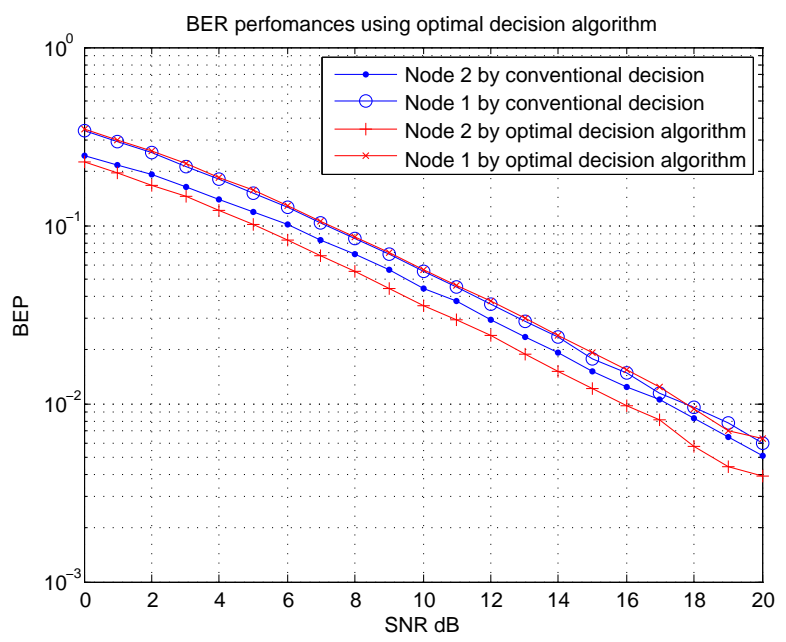

Figure 8. Optimal decision decoding algorithm (ODDA) for an asymmetric TWRC

(ODDA). The simulation results show that the BER decreased by around $1 \mathrm{~dB}$ and the system performance improved. In fact, the proposed algorithm improves spectral efficiency, data rate, capacity and link reliability with minimal cost or complexity.

\section{REFERENCES}

[1] Ahlswede, R. and Ning Cai and Li, S.-Y.R. and Yeung, R.W. Network information flow, Information Theory, IEEE Transactions on, Vol. 46, No. 4, 1204 -1216, 2000.

[2] Siamack Ghadimi. Performance assessment of wireless Two Way Relay Channel systems, Western University. London
Ontario, 2013.

[3] Katti, Sachin and Gollakota, Shyamnath and Katabi, Dina. Embracing wireless interference: analog network coding, SIGCOMM Comput. Commun. Rev., Vol. 37, No. 4, 397-408, 2007.

[4] Zheng Li and Xia, Xiang-Gen and Bin Li. Achieving full diversity and fast ML decoding via simple analog network coding for asynchronous two-way relay networks, Communications, IEEE Transactions on, Vol. 57, No. 12, 3672-3681, 2009.

[5] Peh, E.C.Y. and Ying-Chang Liang and Yong Liang Guan. Power control for physical-layer network coding in fading environments, Personal, Indoor and Mobile Radio Communications, 2008. PIMRC 2008. IEEE 19th International Symposium on, $1-5,2008$.

[6] J. Proakis. Digital Communications, McGrawHills, 2000.

[7] Koike-Akino, T. and Popovski, P. and Tarokh, V. Optimized constellations for two-way wireless relaying with physical network coding, Selected Areas in Communications, IEEE Journal on, Vol. 27, No. 5, 773 -787, 2009.

[8] Darmawan, A. and Kim, S.W. and Hiroyuki Morikawa. Amplify-and-Forward Scheme in Cooperative Spatial Multiplexing, Mobile and Wireless Communications Summit, 16th

IST, 1-5, 2007. 